\title{
Revendo a Orientação Dietética na Gota
}

\section{Diet Orientation on Gout Revisited}

\author{
Geraldo da Rocha Castelar Pinheiro ${ }^{(1)}$
}

\section{RESUMO}

A gota é uma condição médica comum, sendo a principal causa de artropatia inflamatória na população masculina adulta. Os principais fatores envolvidos no aumento da prevalência dessa enfermidade, observados nas duas últimas décadas, incluem aumento da longevidade, uso de diuréticos e aspirina em dose baixa, insuficiência renal crônica, hipertensão arterial sistêmica, obesidade e síndrome metabólica. Entre as causas de hiperuricemia observadas nos pacientes com gota, está a ingestão dietética de purinas. A associação entre dieta hiperprotéica e alimentos ricos em purina e consumo de bebidas alcoólicas com hiperuricemia e gota carecia, até bem pouco tempo, de documentação científica adequada. Com base nos últimos trabalhos sobre o tema, publicados na literatura, este artigo faz uma atualização a respeito da orientação dietética mais adequada para os pacientes de gota.

Palavras-chave: gota, orientação dietética, ingestão alcoólica.

\section{INTRODUÇÃO}

Gota, também chamada de doença por depósito de cristal de urato monossódico, é um tipo de artropatia inflamatória desencadeada pela cristalização do ácido úrico dentro da articulação(1). É uma condição médica comum, sendo a principal causa de artropatia inflamatória na população masculina adulta ${ }^{(2)}$. Em levantamento realizado entre 1988 e 1994, nos Estados Unidos, foi estimado que cerca de 2\% dos homens com mais de 30 anos e das mulheres com mais de 50 anos tinham o diagnóstico de gota ${ }^{(3)}$. Essa prevalência aumenta com o avançar da idade e alcança $9 \%$ dos homens e $6 \%$ das mulheres com mais de 80 anos $^{(4)}$. Os principais fatores envolvidos no aumento da prevalência dessa enfermidade, observados nas duas últimas décadas, incluem aumento da longevidade, uso de diuréticos e aspirina em dose baixa, insuficiência renal crônica, hipertensão arterial sistêmica, obesidade e síndrome metabólica ${ }^{(5)}$.

\begin{abstract}
Gout is a common medical condition, being the main cause of inflammatory arthropathy in adult men. The main factors involved in the increased prevalence of this disease, observed in the two last decades, include: use of diuretics and low dose aspirin, chronic renal insufficiency, hypertension, obesity, and metabolic syndrome. Among the causes of hyperuricemia seen in patients with gout is the dietary ingestion of purines. The association between a high-protein diet, purine-rich foods, and alcoholic beverages with hyperuricemia and gout, until very recently, lack adequate scientific documentation. Based on the most recently publications on this subject, the present article brings an actualization about the most adequate dietary orientation for patients with gout.
\end{abstract}

Keywords: Gout, dietary orientation, alcoholic beverages.

\section{PATOGÊNESE DA GOTA}

O ácido úrico é um ácido fraco (pKa 5,8) que existe largamente como urato, a forma ionizada, em $\mathrm{pH}$ fisiológico ${ }^{(1)}$. A quantidade de urato no organismo é o resultado do balanço entre a ingesta dietética, a síntese endógena e a taxa de excreção (Figura 1). A hiperuricemia pode ser o resultado da redução da excreção de ácido úrico ( $85 \%$ a $90 \%)$ ou do aumento da produção (10\% a $15 \%)$. Mesmo em indivíduos cuja excreção diária de ácido úrico esteja acima do normal (hiperprodutores), pode ocorrer redução relativa na eliminação renal do ácido úrico, ou seja, tanto a hiperprodução quanto a hipoexcreção contribuiriam para a hiperuricemia ${ }^{(1)}$.

Anormalidade no metabolismo das enzimas envolvidas na síntese de nucleotídeos purínicos (por exemplo, deficiência da hipoxantina-guanina fosforibosiltransferase ou hiperatividade da fosforibosilpirofosfato sintetase) é 


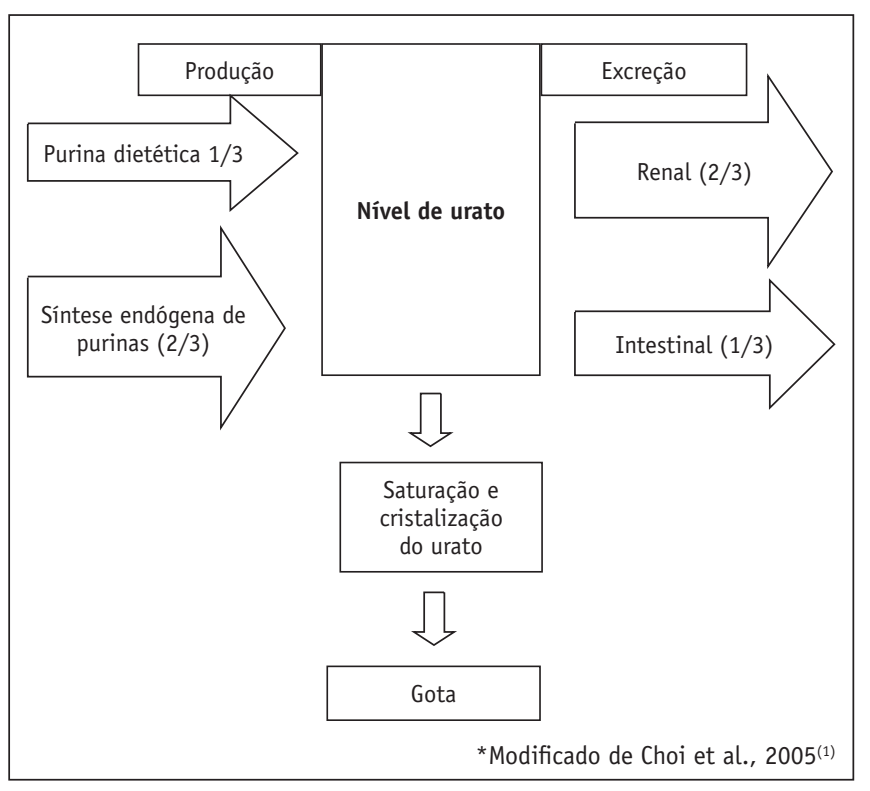

Figura 1 - Patogênese da gota*

identificada em apenas uma pequena fração (menos de $10 \%)$ dos hiperprodutores ${ }^{(6)}$. As outras causas conhecidas de hiperprodução de ácido úrico incluem turnover aumentado dos nucleotídeos (por exemplo, desordens mielo e linfoproliferativas, anemia hemolítica e psoríase), degradação acelerada do trifosfato de adenosina (ATP) (por exemplo, abuso de etanol, doenças do armazenamento de glicogênio, tipos I, III, V e VII, ingestão de frutose, intolerância hereditária à frutose, hipoxemia e hipoperfusão tecidual e exercício muscular excessivo) e ingestão excessiva de purinas na dieta ${ }^{(6)}$.

Em síntese, a causa da gota está relacionada tanto a fatores genéticos quanto ambientais, mas a prevalência crescente dessa enfermidade, observada nas duas últimas décadas, acredita-se ser devida, principalmente, aos fatores ambientais relacionados com os hábitos de vida e com a dieta.

\section{O PAPEL DA DIETA NA HIPERURICEMIA E NA GOTA}

A ingestão dietética de purinas pode contribuir substancialmente para os níveis de ácido úrico do sangue. Em um indivíduo sadio, a instituição de uma dieta isenta de purinas por alguns dias pode reduzir os níveis de ácido úrico no sangue na média de 4,95 mg/dl $(297 \mu \mathrm{mol} / \mathrm{l})$ para $2,95 \mathrm{mg} / \mathrm{dl}(178 \mu \mathrm{mol} / \mathrm{l})^{(7,8)}$. É importante salientar que a biodisponibilidade das purinas presentes em determinado alimento depende tanto do seu conteúdo celular quanto da atividade transcripcional e metabólica dessas células $^{(1)}$. Pouco se sabe, entretanto, sobre a identidade e a quantidade precisa de purinas individuais na maioria dos alimentos, especialmente quando cozidos ou processados industrialmente ${ }^{(9)}$. A adição de purinas dietéticas para pacientes em dietas isentas de purina produz aumento variável da uricemia, na dependência da formulação e da dose das purinas administradas $^{(7)}$. O ácido ribonucléico (RNA), por exemplo, tem um efeito maior que as quantidades equivalentes de ácido desoxirribonucléico (DNA) ${ }^{(10)}$, ribomononucleotídeos têm efeito maior que os ácidos nucléicos e a adenina, maior que a guanina ${ }^{(11)}$.

A associação entre dieta hiperprotéica e alimentos ricos em purina com a hiperuricemia e a gota carecia de documentação científica adequada. Recentemente, um estudo prospectivo (Health Professionals Follow-up Study HPFS) acompanhou 47.150 homens sem história prévia de gota $^{(12,13)}$. Durante os 12 anos do estudo, ocorreram 730 casos incidentes de gota. Homens no quintil mais alto de consumo de carne apresentaram risco $41 \%$ mais elevado de gota quando comparados aos do quintil mais baixo. Da mesma forma, aqueles no quintil mais alto de consumo de frutos do mar apresentaram risco $51 \%$ mais elevado em relação aos do quintil mais baixo. Por outro lado, homens no quintil mais elevado de ingesta de laticínios com baixo teor de gordura apresentaram risco $44 \%$ menor de gota quando comparados àqueles no quintil mais baixo. Importante destacar que o consumo de aveia e de vegetais ricos em purinas (por exemplo, ervilha, feijão, vagem, lentilhas, espinafre, cogumelos e couve-flor) não foi associado a aumento do risco de gota.

Li-Ching et al. já haviam chamado a atenção para o fato de que o consumo de alimentos ricos em purina per se não seja fator de risco importante para o aparecimento de gota. De fato, estudando indivíduos tailandeses que se alimentavam exclusivamente de vegetais e produtos à base de soja, encontraram redução no risco de desenvolver gota ${ }^{(14)}$.

Um outro trabalho recente (Third National Health and Nutrition Examination Survey - NHANES) avaliou 14.809 indivíduos (6.932 homens e 7.877 mulheres) com idade superior a 20 anos, entre 1988 e 1994(15). Buscando examinar a relação entre o consumo de alimentos ricos em purinas, proteínas e laticínios e uricemia, encontrouse associação positiva entre níveis de uricemia e aumento no consumo total de carne e frutos do mar, ocorrendo, entretanto, redução quando do aumento na ingestão de laticínios. 
Nos dois estudos citados, não houve associação entre o consumo total de proteínas e os níveis séricos de ácido úrico ${ }^{(13,15)}$. Embora dietas hiperprotéicas contenham grandes quantidades de purinas, o efeito uricosúrico associado com essas dietas pode, na realidade, reduzir os níveis do ácido úrico sérico ${ }^{(15)}$.

Do ponto de vista prático, esses dados sugerem que, em pacientes com hiperuricemia ou gota, a restrição de purinas dietéticas deve se limitar às purinas de origem animal, mas não às de origem vegetal. Convém enfatizar que vegetais ricos em purinas são excelentes fontes de proteínas, fibras, vitaminas e minerais, sendo essenciais a uma alimentação $\operatorname{adequada}^{(16)}$.

Ainda de acordo com o estudo HPFS, o risco de gota era significativamente maior em homens com índice de massa corporal (IMC) maior ou igual a 25 e a magnitude dessa associação variava positivamente com o aumento do $\mathrm{IMC}^{(17)}$. Indivíduos que haviam engordado $13,6 \mathrm{~kg}$ ou mais tiveram aumento de duas vezes no risco de gota quando comparados com pessoas sem ganho de peso. Por outro lado, indivíduos que tinham emagrecido mais de 4,5 $\mathrm{kg}$ tiveram o risco de gota significativamente diminuído. Nos pacientes com aumento da adiposidade e naqueles com síndrome de resistência à insulina, a hiperuricemia comumente observada é decorrente, também, de redução na uricosúria secundária à insulina ${ }^{(18)}$.

\section{O PAPEL DO CONSUMO DE BEBIDAS ALCOÓLICAS NA GOTA}

Um aspecto de grande importância na orientação dietética do paciente com hiperuricemia ou gota é o consumo de bebidas alcoólicas. A ingestão de álcool aumenta a uricemia por incrementar a degradação do ATP em adenosina monofosfato (AMP), que é rapidamente convertido em ácido úrico ${ }^{(19)}$. Por outro lado, a desidratação e a acidose metabólica, relacionadas com o consumo excessivo de bebidas alcoólicas, reduzem a excreção renal de ácido úrico, contribuindo para a hiperuricemia ${ }^{(20)}$.

Embora o senso comum reconheça a associação positiva entre consumo de álcool e risco de gota, não existiam estudos adequados comprovando-a. Além disso, diferenças potenciais entre os diversos tipos de bebidas alcoólicas e o risco de gota nunca haviam sido analisadas de forma sistemática.

A mesma coorte de 47.150 homens (estudo HPFS), acompanhada por Choi et al. ${ }^{(12)}$, foi investigada para a relação entre consumo de álcool e risco de gota incidente. Para o cálculo do consumo de bebidas alcoólicas, utilizaram-se parâmetros descritos na tabela 1. Como supracitado, durante o período de estudo, 730 casos confirmados de gota foram observados. A tabela 2 ilustra o risco relativo multivariado (RRM) de gota para os diferentes consumos de bebida alcoólica e indivíduos abstêmios para etanol. Encontrou-se a associação positiva entre o consumo de álcool e gota, independente da dieta e de outras causas de hiperuricemia, como idade, IMC, hipertensão arterial, uso de diuréticos e insuficiência renal crônica. Quanto aos diferentes tipos de bebidas alcoólicas, o consumo de cerveja mostrou ser a associação positiva mais forte com o risco de gota, com RRM por garrafa ou lata/dia de 1,49 (IC 95\% 1,32-1,70). O consumo de destilados também foi significativamente associado com gota, RRM por dose/dia de 1,15 (IC 95\% 1,04-1,22). O consumo de vinho, entretanto, não apresentou associação com gota, sendo o RRM por copo de vinho/dia de 1,04 (IC 95\% 0,88-1,22). Os autores concluíram que o consumo de álcool tem associação positiva com o risco de desenvolver gota. A tabela 3 ilustra a variação do risco de gota e o consumo de bebidas alcoólicas. A cerveja confere risco maior que as bebidas destiladas, enquanto a ingesta moderada de vinho não aumenta o risco. Além do seu teor de álcool, a cerveja é rica em purinas, em especial guanosina, favorecendo a hiperuricemia e o risco de $\operatorname{gota}^{(21)}$. Por outro lado, é possível que componentes não-alcoólicos presentes no vinho desempenhem papel relevante no metabolismo do ácido úrico, de sorte que, mesmo se ingerido em quantidades moderadas, não levaria a aumento no risco de $\operatorname{gota}^{(12)}$.

TABELA 1

ParÂMetros UTILIZAdoS NA AVALIAÇÃo DO CONSUMO DE BEBIDAS ALCOÓLICAS ${ }^{(12)}$

\begin{tabular}{lccc}
\hline & Unidade & Volume & Conteúdo de etanol \\
\hline Cerveja & Lata ou garrafa & $355 \mathrm{ml}(12 \mathrm{oz})$ & $12,8 \mathrm{~g}$ \\
Vinho & Copo de vinho & $118 \mathrm{ml}(4 \mathrm{oz})$ & $11,0 \mathrm{~g}$ \\
Destilado & Dose & $44 \mathrm{ml}(1,5 \mathrm{oz})$ & $14,0 \mathrm{~g}$ \\
\hline
\end{tabular}

TABELA 2

Risco RELATIVO MULTIVARIAdO PARA GOTA INCIDENTE, EM RELAÇÃO A HOMENS ABSTÊMIOS, DE ACORDO COM O CONSUMO DIÁRIO (QUANTIDADE) DE BEBIDA ALCOÓLICA ${ }^{(12)}$

\begin{tabular}{lcccc}
\hline Consumo diário & $10-14,9(\mathrm{~g} / \mathrm{dia})$ & $\mathbf{1 5 - 2 9 , 9}(\mathrm{g} / \mathrm{dia})$ & $\mathbf{3 0 - 4 9 , 9}$ (g/dia) & $\geq 50$ (g/dia) \\
\hline Risco relativo & $1,32($ IC $95 \%$ 0,99-1,75) & $1,49($ IC $95 \%$ 1,14-1,94) & 1,96 (IC 95\% 1,48-2,60) & 2,53 (IC 95\% 1,73-3,70) \\
\hline
\end{tabular}


TABELA 3

RisCO RELATIVO MULTIVARIADO PARA GOTA INCIDENTE, EM RELAÇÃO A HOMENS ABSTÊMIOS, DE ACORDO COM O CONSUMO DIÁRIO (TIPO) DE BEBIDA ALCOÓLICA ${ }^{(12)}$

\begin{tabular}{|c|c|c|c|}
\hline $\begin{array}{l}\text { Tipo de } \\
\text { bebida }\end{array}$ & $\begin{array}{c}\text { Cerveja } \\
\text { lata/garrafa }(355 \mathrm{ml})\end{array}$ & $\begin{array}{c}\text { Destilado } \\
\text { dose }(44 \mathrm{ml})\end{array}$ & $\begin{array}{c}\text { Vinho } \\
\text { copo }(118 \mathrm{ml})\end{array}$ \\
\hline & $\begin{array}{c}1,49 \\
\text { (IC } 95 \% \text { 1,32-1,70) }\end{array}$ & $\begin{array}{c}1,15 \\
\text { (IC } 95 \% \text { 1,04-1,22) }\end{array}$ & $\begin{array}{c}1,04 \\
\text { (IC 95\% 0,88-1,22) }\end{array}$ \\
\hline
\end{tabular}

\section{CONSIDERAÇÕES FINAIS}

$\mathrm{O}$ atendimento médico consciente de um paciente com gota é um desafio na prática reumatológica. Assim como lidar com as queixas álgicas de uma paciente com fibromialgia ou artrite reumatóide, consultar um paciente com gota exige, além do conhecimento médico, a necessidade de ver essa pessoa em um contexto biopsicossocial muito particular. Diferentemente das mulheres, homens costumam ir menos ao médico, não possuem uma cultura de prevenção de doenças e, freqüentemente, negam suas fraquezas e limitações. Além do grau de conhecimento sobre sua doença e seu tratamento ser muito baixo, a adesão dos pacientes com gota é das mais baixas na prática ambulatorial reumatológica ${ }^{(22,23)}$. Associado a isso, nossa cultura valoriza o consumo abundante de comida e pouco faz para desestimular o exagero em bebidas alcoólicas. Identificar e abordar esses aspectos com o paciente exige perícia e sensibilidade por parte do médico.

\section{REFERÊNCIAS}

1. Choi HK, Mount DB, Reginato AM: Pathogenesis of gout. Ann Intern Med 143: 499-516, 2005.

2. Terkeltaub RA: Gout. N Engl J Med 349: 1647-55, 2003.

3. Kramer HM, Curhan G: The association between gout and nephrolitiasis: the National Health and Nutrition Examination Survey III, 1988-1994. Am J Kidney Dis 40: 37-42, 2002.

4. Arromdee E, Michet CJ, Crowson CS, O'Fallow WM, Gabriel SE: Epidemiology of gout: is the incidence rising? J Rheumatol 29: 2403-6, 2002.

5. Bieber J, Terkeltaub RA: Gout: on the brink of novel therapeutic options for an ancient disease. Arthritis Rheum 50: 2400-14, 2004

6. Terkeltaub RA: Gout: fresh insights into an ancient disease. Science \& Medicine 3: 22-31, 1996.

7. Griebsch A, Zollner N: Effect of ribomononucleotides given orally on uric acid production in man. Adv Exp Med Biol 41: 443-9, 1974.

8. Coe FL, Moran E, Kavalich AG: The contribution of dietary purine overconsumption to hyperuricemia in calcium oxalate stone formers. J Chronic Dis 29: 793-800, 1976.
A hiperuricemia e a gota, geralmente, estão associadas com obesidade, hipertensão, dislipidemia, aterosclerose e intolerância à glicose (síndrome metabólica). Atender um paciente com gota representa oportunidade singular para uma avaliação metabólica mais extensa e uma orientação dietética mais abrangente. Assim sendo, a orientação médica deve contemplar mudanças de hábitos de vida e informações precisas sobre dieta e consumo de bebidas alcoólicas.

Quando presentes, tanto o tabagismo quanto o sedentarismo devem ser evitados. A orientação dietética deve ter como prioridades o controle do peso corporal, da pressão arterial sistêmica e das eventuais alterações metabólicas presentes, como a intolerância à glicose e a dislipidemia.

Quanto à hiperuricemia propriamente dita, é suficiente enfatizar a necessidade de se restringir alimentos ricos em purinas de origem animal (carnes, miúdos, embutidos e frutos do mar). Alimentos ricos em purinas de origem vegetal não precisam nem devem ser evitados e o consumo de laticínios com baixo teor de gordura deve ser estimulado.

É importante "negociar" com o paciente que faz uso de bebida alcoólica quanto ao momento de consumi-la, a quantidade e o tipo de bebida. Fazer uso de bebidas alcoólicas durante ou logo após um episódio agudo pode prolongá-lo ou reduzir o intervalo de recidiva. Após o controle adequado de uma crise aguda, a ingestão de uma quantidade moderada (por exemplo, uma taça de vinho ao dia) pode ser permitida. O uso de cerveja, principalmente em grandes quantidades, deve ser formalmente evitado.

9. Gibson T, Rodgers AV, Simmonds HA, Court-Brown F, Todd E, Meilton V: A controlled study of diet in patients with gout. Ann Rheum Dis 42: 123-7, 1983.

10. Zollner N, Griebsch A: Diet and gout. Adv Exp Med Biol 41: 435-42, 1974.

11. Clifford AJ, Riumallo JA, Young VR, Scrimshaw NS: Effects of oral purines on serum and urinary uric acid of normal, hyperuricemic and gouty humans. J Nutr 106: 428-50, 1976.

12. Choi HK, Atkinson K, Karlson EW, Willett W, Curhan G: Alcohol intake and risk of incident gout in men: a prospective study. The Lancet 363: 1277-81, 2004.

13. Choi HK, Atkinson K, Karlson EW, Willet WC, Curhan G: Purine-rich foods, dairy and protein intake, and the risk of gout in men. N Engl J Med 350: 1093-103, 2004.

14. Li-Ching L, Chi-Yin H, Ching-Ying Y, Meei-Shyuan L, Su-Hua H, Ching-Lan C: A case-control study of the association of diet and obesity with gout in Taiwan. Am J Clin Nutr 78: 690-701, 2003.

15. Choi HK, Simin L, Curhan G: Intake of purine-rich foods, protein, and dairy products and relationship to serum levels of uric acid. The Third National Health and Nutrition Examination Survey. Arthritis Rheum 52: 283-9, 2005. 
16. Willer WC, Stampfer MJ: Rebuilding the food pyramid. Sci Am 288:64-71, 2003.

17. Choi HK, Atkinson K, Karlson EW, Willet WC, Curhan G: Obesity, weight change, hypertension, diuretic use, and the risk of gout in men: the Health Professionals Follow-up Study. Arch Intern Med 165:742-8, 2005.

18. Yamashita S, Matsuzawa Y, Tokunaga K, Fujioka S, Tarui S: Studies on the impaired metabolism of uric acid in obese subjects: marked reduction of renal urate excretion and its improvement by a low-calorie diet. Int J Obes 10: 255-64, 1986.

19. Puig JG, Fox IH: Ethanol-induced activation of adenine nucleotide turnover. Evidence for a role of acetate. J Clin Invest 74:936-41, 1984.
20. Lieber CS, Jones DP, Losowsky MS, Davidson CS: Interrelation of uric acid and ethanol metabolism in man. J Clin Invest 41: 1863-70, 1962.

21. Gibson T, Rodgers AV, Simmonds HA, Toseland P: Beer drinking and its effects on uric acid. Br J Rheumatol 23: 203-9, 1984.

22. Pinheiro GRC, Andrade CAF, Conceição LHR, Bomfim MG, Klumb EM, Levy RA: Avaliação do grau de conhecimento sobre a doença e terapêutica nos pacientes com artrite gotosa. Rev Bras Reumatol 36: 196, 1996.

23. Teixeira IC, Pinheiro GRC, Silva GB, Gayer CRM, Freire SM: Estudo da aderência ambulatorial de pacientes com gota. Rev Bras Reumatol 40(Supl 1): 100, 2000. 